\title{
08 IDENTIFYING RESIDENCES AT INCREASED RISK OF EXPERIENCING AN UNINTENTIONAL HOUSE FIRE INCIDENT, DEATH OR INJURY
}

doi:10.1136/injuryprev-2012-040590c.8

SL Turner, SE Rodgers, RA Lyons. Swansea University, UK

Background Although a large body of literature exists for individual-level risk factors associated with fire-related death and injury; few studies have explored household-level risk factors, or distinguished risk factors for different house fire outcomes.

Aims/Objectives/Purpose To use anonymised routinely collected records, to identify distinguishing household-level risk factors for experiencing an accidental house fire incident, death or injury.

Methods A household-level, matched case-control study. Case households were identified using Fire and Rescue Service (FRS) records, and matched to control households, on fire date and FRS region. The Secure Anonymised Information Linkage databank, which holds anonymised routinely collected demographic, health, education and environment records; will be used to identify household-level predictor variables. Logistic regression and classification and regression tree analysis will be used to identify risk factors.

Results/Outcomes 7027 case households were matched to 351350 control households. Residents were identified using the Welsh Demographic Service; a dataset containing details of all individuals registered with a General Practice. Household level predictor variables to be investigated: household demographics, property characteristics, area level deprivation, number of residents who smoke, have mental health conditions, special education needs, special medical requirements and limited mobility. Subanalyses will explore risk factors associated with different house fire outcomes. Significance/Contribution to the Field This study is one of the first to use anonymised routinely collected records, on a large scale, to identify household-level risk factors for experiencing an unintentional house fire. These findings will help the FRS to target home fire safety interventions more effectively. 